\title{
Hématomes Extraduraux Chez L'adulte Au Togo
}

Katanga Anthony Beketi

Service de Neurochirurgie, CHU Sylvanus Olympio, Lomé, Togo

Edem Yaovi James

Service d'orthopédie traumatologie, CHU Sylvanus Olympio, Lomé, Togo

Faré Gnadi-Piou

Service d'orthopédie traumatologie, CHU Kara, Kara, Togo

Essossinam Kpelao

Pazimassouwé Tagba

Abdel-kader Moumouni

K. M Hobli Ahanogbé

Service de Neurochirurgie, CHU Sylvanus Olympio, Lomé, Togo

Atchi Walla

Assang Michel Dossim

Service d'orthopédie traumatologie, CHU Sylvanus Olympio, Lomé, Togo

Doi: 10.19044/esj.2018.v14n6p229 URL:http://dx.doi.org/10.19044/esj.2018.v14n6p229

\section{Abstract}

Purpose: The purpose of this work was to evaluate our neurosurgical practice about extradural hematoma and to describe their prognosis in our context. Patients and methods: This is a retrospective study of patients treated for extradural hematoma at Sylvanus Olympio university teaching hospital, which houses the only neurosurgery unit in Togo between April 2008 and August 2014. We included in our study, the files of patients of both sexes, aged over 15 years, with extradural hematoma isolated or not at the cerebral scanner, treated during the study period. Thus, 62 patients were taken into account. There was a male predominance (sex ratio of 19.7). The average age was 29.8 years with extremes of 02 and 70 years of which $41.9 \%$ of patients were between 20 and 29 years old. The surgical technique was the making of a burr hole or a cranial flap. We examined the parameters related to surgical treatment and the evolutionary mode. Results: In this study, 34 patients (54.8\%) underwent surgery: cranial section (27 cases), widened hole craniotomy (07 cases). The average time between trauma and surgery was 09 days with extremes of 01 to 30 days. Twenty-four patients were operated more than 48 hours after diagnosis and the average postoperative follow-up time was 11.1 days with extremes of 07 and 18 days. 
Twenty-eight patients (45.2\%) were not operated on. Among them, 20 patients presented with a HED blade (thickness of less than 10mm) and the eight (08) others for lack of financial means. The average hospital follow-up time for non-operated patients was 15.4 days with extremes of 08 and 60 days. The evolution was evaluated by the Glasgow Outcome Scale over three months. Fifty-five patients or $88.7 \%$ recovered without sequel. We obtained 3 cases $(4.8 \%)$ of deaths including 2 cases that were not operated due to lack of financial means. Conclusion: We have observed that a low Glasgow admission score, long treatment delays, and associated lesions are factors that appear to reduce the prognosis in the treatment of extradural hematoma in Togo. These prognostic factors must be better documented in our future studies to identify them in order to act on them in order to reduce the morbidity and mortality that these extra-mural hematoma cause in our environments.

Keywords: Extradural hematoma, evacuation, prognostic factors, Togo

\section{Résumé}

Objectif: Le but de ce travail a été d'évaluer notre pratique neurochirurgicale concernant les hématomes extraduraux (HED) et d'en décrire leurs pronostics dans notre contexte. Patients et Méthodes : Il s'agit d'une étude rétrospective portant sur les dossiers de patients traités pour hématome extradural au CHU Sylvanus Olympio (CHU-SO) qui abrite l'unique service de neurochirurgie au Togo, entre avril 2008 et août 2014. Nous avons inclus dans notre étude, les dossiers de patients des deux sexes, âgés de plus de 15 ans, présentant un hématome extradural isolé ou non au scanner cérébral, pris en charge pendant la période d'étude. Ainsi, 62 patients ont été pris en compte. Il y avait une prédominance masculine (sexe ratio de 19,7). L'âge moyen était de 29,8 ans avec des extrêmes de 02 et 70 ans dont $41,9 \%$ de patients avaient entre 20 et 29 ans. La technique chirurgicale a été la réalisation d'un trou de trépan ou un volet crânien. Nous avons examiné les paramètres liés au traitement chirurgical et le mode évolutif. Résultats : Dans cette étude, 34 patients $(54,8 \%)$ ont bénéficié d'une intervention chirurgicale : volet crânien ( 27 cas), craniotomie par trou de trépan élargi (07 cas). Le délai moyen entre le traumatisme et l'intervention chirurgicale était de 09 jours avec des extrêmes de 01 et de 30 jours. Vingt-quatre (24) patients ont été opérés plus de $48 \mathrm{~h}$ après le diagnostic et le délai moyen de suivi post opératoire était de 11,1 jours avec des extrêmes de 07 et de 18 jours. Vingt-huit patients $(45,2 \%)$ n'ont pas été opérés. Parmi eux, 20 patients présentaient une lame d'HED (épaisseur de moins de $10 \mathrm{~mm}$ ) et les huit (08) autres par manque de moyens financiers. Le délai moyen de suivi hospitalier des patients non opérés était de 15,4 jours avec des extrêmes de 08 et 60 jours. L'évolution a été évaluée par le Glasgow Outcome Scale sur trois mois. Cinquante-cinq patients 
soit $88,7 \%$ ont récupéré sans séquelles. Nous avons obtenu 3 cas $(4,8 \%)$ de décès dont 2 cas qui n'ont pas été opérés par manque de moyens financiers. Conclusion: Nous avons observé qu'un score de Glasgow bas à l'admission, les délais de prise en charge longs et les lésions associées sont des facteurs qui semblent dégrader le pronostic dans le traitement des hématomes extraduraux au Togo. Ces facteurs pronostiques doivent être mieux documentés dans nos futures études afin de les identifier afin d'agir sur eux dans le but de réduire la morbi-mortalité que ces hématomes extraduraux occasionnent dans nos milieux.

Mots-clés : Hématome extradural, trépanation, facteurs pronostiques, Togo

\section{Introduction}

Les traumatismes cranio-encéphaliques constituent un problème important de santé publique et engendrent jusqu'à $50 \%$ de décès chez les traumatisés et de handicaps permanents avec soins continus chez les survivants (KIBOI, 2011). Ce problème se pose avec plus d'acuité dans les pays en développement en raison de l'irrespect des normes de sécurité routière et sur les lieux de travail, du mauvais état des routes et des engins roulants puis du déficit de sécurité sociale (GAYE, 2010 ; HYDER, 2007).

Les hématomes extra duraux qui résultent de ces traumatismes crâniens sont une complication grave. Seul le traitement précoce permet la réduction de la morbi-mortalité liée à ces hématomes.

$\mathrm{Au}$ Togo, tous ces patients sont orientés vers notre service qui est le seul centre de neurochirurgie du pays.

En entreprenant ce travail, notre but a été d'évaluer notre pratique neurochirurgicale concernant ces hématomes extraduraux et d'en décrire leurs pronostics dans notre contexte.

\section{Méthodologie}

\section{Contexte et population d'étude}

Il s'agit d'une étude rétrospective portant sur les dossiers des patients présentant un hématome extradural, pris en charge au CHU Sylvanus Olympio (CHU-SO) qui abrite l'unique service de neurochirurgie au TOGO, entre le $1^{\text {er }}$ avril 2008 et le 30 août 2014. Ce service a été créé au Togo en 2008. Une équipe de trois (3) neurochirurgiens était responsable des astreintes hebdomadaires pour la prise en charge spécialisée. La totalité de la prise en charge, pré et péri-opératoire, était à la charge financière du patient. Les deux salles opératoires n'étaient pas des salles exclusivement destinées à la neurochirurgie. 


\section{Sélection des patients}

Nous avons inclus dans notre étude, les dossiers de patients des deux sexes, âgé de plus de 15 ans, présentant un hématome extradural isolé ou non au scanner cérébral, traité pendant la période d'étude. Nous n'y avons pas inclus les patients enregistrés et dont les dossiers médicaux n'ont pas été retrouvés ainsi que les traumatisés crâniens non explorés par tomodensitométrie (TDM) cérébrale.

Ainsi, 62 patients ont été pris en compte. Il y avait une prédominance masculine (sexe ratio de 19,7). L'âge moyen était de 29,8 ans avec des extrêmes de 02 et 70 ans dont $41,9 \%$ de patients avaient entre 20 et 29 ans.

\section{Technique chirurgicale}

Pour les patients opérés, la technique à consister à réaliser soit un volet crânien ou un trou de trépan afin d'évacuer l'hématome.

\section{Analyse}

Nous avons examiné les paramètres liés au traitement chirurgical et le mode évolutif.

\section{Résultats}

Dans cette étude, 34 patients $(54,8 \%)$ ont bénéficié d'une intervention chirurgicale : volet crânien (27 cas), craniotomie par trou de trépan élargi (07 cas). Le délai moyen entre le traumatisme et l'intervention chirurgicale était de 09 jours avec des extrêmes de 01 à 30 jours (Tableau 1). Vingt-quatre patients ont été opérés plus de $48 \mathrm{~h}$ après le diagnostic et le délai moyen de suivi post opératoire était de 11,1 jours avec des extrêmes de 07 et 18 de jours. Tableau 1: Répartition des patients $(n=34)$ en fonction du délai séparant le traumatisme du traitement chirurgical.

\begin{tabular}{lll}
\hline Délai & Effectif & Pourcentage \\
\hline < à 24 heures & 0 & 0,0 \\
24 à 48 heures & 13 & 38,2 \\
48 à 72 heures & 3 & 8,8 \\
3 à 5 jours & 5 & 14,7 \\
6 à 10 jours & 5 & 14,7 \\
11 à 20 jours & 6 & 17,6 \\
21 à 30 jours & 2 & 6,0 \\
Total & 34 & 100,0 \\
\hline
\end{tabular}

Vingt-huit patients $(45,2 \%)$ n’ont pas été opérés. Parmi eux, 20 patients présentaient une lame d'HED (épaisseur de moins de $10 \mathrm{~mm}$ ) et les huit(08) autres par manque de moyens financiers. Le délai moyen de suivi hospitalier des patients non opérés était de 15,4 jours avec des extrêmes de 08 et 60 jours (Tableau 2). Le patient ayant été hospitalisé pendant 60 jours présentait des contusions oedémato-hémorragiques multifocales 
hémisphériques homolatérales associées à une lame d'hématome extradural.

Tableau 2 : Répartition des patients non opérés $(n=28)$ en fonction de la durée du suivi

\begin{tabular}{lll}
\hline Délai & Effectif & Pourcentage \\
\hline 8 à 10 jours & 12 & 42,9 \\
11 à 15 jours & 9 & 32,1 \\
16 à 20 jours & 5 & 17,8 \\
21 à 25 jours & 1 & 3,6 \\
26 à 60 jours & 1 & 3,6 \\
Total & 28 & 100,0 \\
\hline
\end{tabular}

L'évolution a été évaluée par le Glasgow Outcome Scale sur trois mois. Cinquante-cinq patients soit $88,7 \%$ ont récupéré sans séquelles. Nous avons obtenu 3 cas $(4,8 \%)$ de décès dont 2 cas qui n'ont pas été opérés par manque de moyens financiers (Tableau 3 ).

Tableau 3 : Répartition des patients $(n=62)$ en fonction du Glasgow Outcome Scale

\begin{tabular}{lll}
\hline Score & Effectif & Pourcentage \\
\hline Grade 1 : récupération/séquelles minimes & 55 & 88,7 \\
Grade 2 : séquelles mais indépendantes & 4 & 6,5 \\
Grade 3 : conscient mais non autonome & 0 & 0,0 \\
Grade 4 : état végétatif persistant & 0 & 0,0 \\
Grade 5 : décès & 3 & 4,8 \\
Total & 62 & 100,0 \\
\hline
\end{tabular}

\section{Considérations éthiques}

Cette étude a reçu l'aval du comité d'éthiques du CHU Sylvanus Olympio et les auteurs ne déclarent aucun conflit d'intérêt.

\section{Discussion}

Cette étude en dépit de ses limites qui sont propres aux travaux de ce type, permet néanmoins de faire le point sur le traitement et le pronostic des hématomes extraduraux dans le seul service de neurochirurgie de notre pays. L'environnement de pratique de la neurochirurgie dans un pays en développement comme le nôtre impacte sur les résultats de prise en charge (EMEJULU, 2014; FATIGBA, 2015). Les facteurs tels que, l'état embryonnaire des services de neurochirurgie, l'insuffisance de neurochirurgiens, le système limite même de l'organisation des soins et l'absence de sécurité sociale sont autant d'aspérités qui affectent l'administration de soins spécialisés de qualité.

Dans notre travail, nous avons noté non seulement un retard d'admission mais aussi un long délai de prise en charge, surtout des cas relevant pour lesquels il y avait une indication chirurgicale. Seulement 10 patients sur les 34 opérés, l'ont été en situation d'urgence réellement. Plusieurs raisons semblent déterminer ce résultat, à savoir, l'absence de bloc opératoire de neurochirurgie autonome et indépendant, le fait que l'ensemble de consommables au bloc sont à la charge des accompagnants du patient et 
dans une certaine mesure les troubles de conscience sont généralement modérés dans notre étude ( $17,7 \%$ de coma d'emblée). Ibahioin K, retrouvait un délai opératoire moyen de $1 \mathrm{~h} 24$ min pour des patients admis pour HED dans les 48 premières heures ; il s'agit du délai entre l'admission au centre de santé et l'intervention chirurgicale (IBAHIOIN, 2016).

Le nombre élevé de nos patients non opérés $(45,2 \%)$ est surtout justifié par la présentation radio clinique. La plupart présentait une lame d'hématome extradural. Ce chiffre est plus élevé que dans la plupart des séries (BRICOLO, 1984 ; MAAS, 2007 ; WESTER, 1999). Il faut néanmoins constaté que huit patients n'ont pas été opérés, faute de moyens financiers et parmi eux deux sont décédés. Il s'agit de décès évitables qui doivent faire repenser l'organisation du système de santé au plan national.

Le score de Glasgow bas a été surtout trouvé chez des patients dont le pronostic vital a été mauvais. De nombreux auteurs ont signalé qu'il existe une corrélation hautement significative entre le résultat et le score GCS à l'admission (HASELSBERGER, 1988 ; KLUN, 1984 ; KOC,1997.

La mortalité était de 4,8\%, un taux relativement faible par rapport à des centres apparemment mieux organisés. Elle est de 17,77\% pour les HED opérés à Casablanca en raison de lésions associées plus importantes corrélées à des traumatismes plus violents, une caractéristique des grandes agglomérations (IBAHIOIN, 2016). Au vue des délais d'admission longs, on est en droit de s'interroger sur la proportion de patients décédés des suites d'HED sur le lieu du traumatisme ou avant leur prise en charge pour un SAMU inexistant. Le taux de mortalité réel des HED est proportionnellement lié au délai de prise en charge chirurgicale et à la gravité des lésions intracrâniennes associées (TIAN, 2008).

Quant au délai opératoire, des auteurs ont documenté qu'il est d'une importance pronostique chez les patients gravement blessés (HASELSBERGER, 1988 ; SAKAS, 1995 ; SEELIG, 1981). En effet, Kiboi JG a rapporté que les patients dont les hématomes ont été évacués dans les 24 heures présentaient $61,7 \%$ de récupération et $15,9 \%$ d'incapacité modérée contre $17,5 \%$ et $8,8 \%$ de ceux opérés plus de 4 jours après un traumatisme (KIBOI, 2011).

Le seul cas de décès post opératoire de notre travail, présentait des lésions multiples intracrâniennes et la prise en charge chirurgicale a été tardive.

Nous avons observé qu'un score de Glasgow bas à l'admission, les délais de prise en charge longs et les lésions associées sont des facteurs qui semblent dégrader le pronostic dans le traitement des hématomes extraduraux au Togo. Des travaux effectués en Afrique subsaharienne l'ont noté (KIBOI, 2011). Ces facteurs pronostiques doivent être mieux documentés dans nos 
futures études afin de les identifier et d'agir sur eux dans le but de réduire la morbi-mortalité que ces hématomes extraduraux occasionnent dans nos milieux.

\section{References:}

1. BRICOLO A, PASUT L. (1984). Extadural hematoma toward zero mortality. Neurosurgery. 14: 8-12.

2. EMEJULU JK, UCHE EO, NWANKWO EU. (2014). The challenges of managing acute extradural hematoma in a Nigerian neurosurgical center still a long way to go. World Neurosurg. Dec;82(6):969-73.

3. FATIGBA HO, SAVI DE TOVE MK, TCHAOU BA, MENSAH E, ALLODE AS, PADONOU J. (2013). Surgical management of head trauma: problems, results, and perspectives at the departmental teaching hospital of Borgou, Benin. World Neurosurg. Sep-Oct;80(34):246-50.

4. GAYE M, DIATTA B, NDOYE N, THIAM AB, DIOP AA, SAKHO Y, BADIANE SB. (2010). Prise en charge des hématomes extraduraux à Dakar à propos de 40 cas. Afr J of Neur Sc.29(1):47-56.

5. HASELSBERGER K, PUCHER R, AUER LM. (1988). Prognosis after acute subdural or epidural hemorrhage. Acta Neurochir (Wien) 90:111-116.

6. HYDER AA, WUNDERLICH CA, PUVANACHANDRA P, GURURAJ G, KOBUSINGYE OC. (2007). The impact of traumatic brain injuries: a global perspective. Neuro Rehabilitation; 22:341-353.

7. IBAHIOIN K, AHANOGBE KMH, DIANKA MB et coll. (2016). Mortalité des hématomes extra duraux à Casablanca en 2013: influence du délai de prise en charge. Médecine du Maghreb. 236:1523.

8. KIBOI JG, KITUNGUU PK, ANGWENYI P, MBUTHIA F, SAGINA LS. (2011). Predictors of functional recovery in African patients with traumatic intracranial hematomas. World Neurosurg. May-Jun;75(5-6):586-91.

9. KLUN B, FETTICH M. (1984). Factors influencing the outcome in acute subdural hematoma. A review of 330 cases. Acta Neurochir (Wien) 71:171-178.

10. KOC RK, AKDEMIR H, OKTEM IS, MERAL M, MENKÜ A. (1997). Acute subdural hematoma: outcome and outcome prediction. Neurosurg Rev 20:239-244.

11. MAAS AI, STEYERBERG EW, BUTCHER I, DAMMERS R, LU J, MARMAROU A et al. (2007). Prognostic value of computerized tomography scan characteristics in traumatic brain injury: results from the IMPACT study. J Neurotrauma; 24:303-314. 
12. SAKAS DE, BULLOCK MR, TEASDALE GM. (1995). One-year outcome following craniotomy for traumatic hematoma in patients with fixed dilated pupils. $J$ Neurosurg 82:961-965.

13. SEELIG JM, BECKER DP, MILLER JD, GREENBERG RP, WARD JD, CHOI SC. (1981). Traumatic acute subdural hematoma: major mortality reduction in comatose patients treated within four hours. $N$ Engl J Med 304:1511-1518.

14. TIAN HL, CHEN SW, XU T, HU J, RONG BY, WANG G, GAO WW, CHEN H. (2008). Risk factors related to hospital mortality in patients with isolated traumatic acute subdural hematoma: analysis of 308 patients undergone surgery. Chin Med J (Engl) 121:1080-1084.

15. WESTER K. (1999). Decompressive surgery for pure epidural hematomas: does neurosurgical expertise improve the outcome? Neurosurgery. 44(3): 495-500. 